\title{
Ultrasonography to Assess the Efficacy of Osteopathic Manipulative Treatment for Lumbar Spine Asymmetry
}

Jeffrey Winter, OMS III; Andrew Kimber, OMS III; Stuart Montenegro, OMS II; Jing Gao, MD

From the College of Osteopathic Medicine at Rocky Vista University in Ivins, Utah.

Financial Disclosures: None reported.

Support: This research was

funded by an Intramural

Research Grant from

Rocky Vista University in 2017 (grant number 2017-0023).

Address correspondence to Andrew Kimber, OMS III, Rocky Vista University College of Osteopathic Medicine, 255 E. Center St, Ivins, UT 84738-6790.

Email: andrew.kimber@rvu.edu

February 10, 2020.
Context: The effectiveness of osteopathic manipulative treatment (OMT) on the lumbar spine has been studied qualitatively, but quantitative measurement of the effects of OMT has not been thoroughly investigated.

Objective: To quantitatively measure the palpated improvements of OMT on the lumbar spine using ultrasonography (US) and correlate palpatory diagnosis with US measurements of lumbar asymmetry.

Methods: From September to November 2018, we recruited 20 adult participants 18 years of age or older. Lumbar somatic dysfunction (SD) was identified via osteopathic palpation. US was then performed on all participants with standard machine settings (frequency, $7 \mathrm{MHz}$; depth, $7 \mathrm{~cm}$; dynamic range, 60; tissue harmonic imaging; and single-image focus). Longitudinal images of each lumbar transverse process were recorded and saved bilaterally by an experienced radiologist and a medical student. The participant's SD was then managed using OMT, including Still technique, myofascial release, muscle energy technique, high-velocity low-amplitude technique, functional positional release, balanced ligamentous tension, and counterstrain. Following OMT, US was performed again in the same method. Measurements of the saved US images were reviewed using a Digital Imaging and Communications in Medicine viewer. These measurements were obtained by 3 separate observers (J.W., A.K., S.M.), using the same computer software. Statistical analysis included a 2-tailed paired $t$-test to analyze rotational asymmetry pre- and posttreatment, an intraclass correlation coefficient (ICC) to test intra- and interobserver reliability, and a Pearson correlation coefficient (PCC) to analyze the correlation between US findings and OMT.

Results: The difference in soft tissue thickness before and after OMT was significant $(P=.014)$, indicating improvements in rotational asymmetry. Side-bending asymmetry did not demonstrate statistically significant improvement. US findings correlated with the physician's palpatory rotational diagnosis ( $\mathrm{PCC}=0.62, P=.004)$. ICC was greater than 0.9 for intra- and interobserver reliability tests of both US operation and offline image processing.

Conclusion: The results of this study demonstrate that US is a feasible method of evaluating the efficacy of OMT. These results show good intra- and interobserver reliability of US acquisition and landmark measurement. Our study suggests that US assessment correlated closely with palpatory diagnosis. Our results also suggest that OMT can significantly improve lumbar rotational asymmetry, but did not improve side-bending asymmetry.

J Am Osteopath Assoc. 2020;120(11):761-769. Published online October 6, 2020. doi:10.7556/jaoa.2020.127

Keywords: low back pain, osteopathic manipulative treatment, somatic dysfunction, ultrasonography 


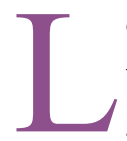

ow back pain (LBP) is a common problem that affects an increasing number of people globally. ${ }^{1}$ LBP management is challenging for several reasons, including varying origins, variable patient pain threshold, and chronic nature. Evaluating the effectiveness of management approaches can be even more difficult because of a lack of quantitative measures.

Compared with pharmaceutical management and invasive procedures, osteopathic manipulative treatment (OMT) is considered both cost-efficient and effective. ${ }^{2-4}$ Typically, the patient's perception of pain and palpatory examination are tools used to diagnose, treat, and measure improvement when using OMT. Pain is a functional parameter to determine the efficacy of a treatment clinically; however, it is less helpful when determining how the treatment caused improvement in the patient's symptoms. Pain is a subjective assessment that depends on a variety of uncontrolled variables. Furthermore, a self-reported perception of pain rated from 1 to 10 is an inconsistent predictor of good clinical outcomes. ${ }^{5}$ With a lack of consistent pain measurement, it can be difficult for osteopathic physicians to consistently and accurately correlate bony landmarks with a clinically significant diagnosis and OMT. ${ }^{6-8}$

Other parameters may provide more consistent results when evaluating OMT. For example, several studies $^{9-12}$ have evaluated the effectiveness of OMT using certain cytokines, pain tolerance, and muscle activation as indicators. Using these nonquantitative assessments, previous studies ${ }^{9-12}$ were able to show that OMT could provide measurable improvement. However, while these results suggested improvements in patients' conditions, they did not speak to the underlying causes of that improvement. The specific mechanism of OMT is still being investigated, ${ }^{13-14}$ and one proposed mechanism, the biomechanical model, theorizes that somatic dysfunction is a result of asymmetry. ${ }^{15}$ Savarese et al $^{16}$ provided support for the biomechanical model by stating that "a somatic dysfunction can present as tissue texture changes, asymmetry, restriction, and tenderness." The biomechanical model is the basis for palpatory examinations, but because of inaccuracies in the identification of landmarks, better measurement techniques are needed.

Imaging techniques that have been used for the assessment of musculoskeletal (MSK) structures include plain film radiography, computed tomography (CT), magnetic resonance imaging (MRI), and ultrasonography (US). Radiography and CT expose the patient to ionizing radiation, MRI is expensive, and these techniques require costly equipment and training to operate. US is inexpensive, radiation-free, and mobile, which makes it a valid option to measure musculoskeletal structures. Previously, US was found to accurately identify lumbar spine landmarks. ${ }^{17}$ It was also used to assess the effects of OMT, including general OMT to the sacral region ${ }^{18}$ and high-velocity, low-amplitude (HVLA) treatment to the lumbar spine. ${ }^{19}$ To influence change in the targeted dysfunction, many OMT practitioners use numerous manipulation techniques for multiple related areas.

Our aim in this study was to assess the feasibility of using US to quantitatively measure asymmetry in the lumbar spine before and after management with multiple OMT techniques.

\section{Methods}

The study protocol was approved by Rocky Vista University institutional review board, and participants provided written informed consent. The study was also funded by an Intramural Research Grant from Rocky Vista University (grant number 2017-0023).

\section{Participants}

We recruited volunteers older than 18 years from the medical school and local community via fliers, emails, and personal invitation from other participants. All participants were asymptomatic at presentation. All participants received a diagnosis, measurements, and OMT. Individuals were excluded if they demonstrated contraindications to receiving OMT (including lack of 
somatic dysfunction) or were not able to provide written informed consent.

\section{Palpatory Assessment}

To assess lumbar spine somatic dysfunction, osteopathic physicians (J.P., J.C, C.E., K.B., A.O., who are noted in the Acknowledgments section), who were assisted by third-year osteopathic medical students (S.M., J.W., A.K.), palpated the participant's lumbar spine. Bony landmarks and the lumbar vertebrae with the most substantial somatic dysfunction were identified, and a diagnosis was determined and recorded by the assessing physician (J.P., J.C., C.E., K.B., who are noted in the Acknowledgments section) to be used in creating (J.P., J.C, C.E., K.B.) a management plan. The vertebrae was palpated and diagnosed in 3 planes of motion: flexion/ extension, rotation, and side-bending. ${ }^{20}$ This vertebrae was then marked by the physician (J.P., J.C, C.E., K.B.) with a felt-tip pen to be used as a landmark, not a definitive point of measurement. The observers who performed US (J.G. and A.K.) were blinded to the osteopathic palpatory examination results. Lumbar somatic dysfunction could be related to dysfunction in biomechanically related areas. Other biomechanically-related structures, including the sacrum, pelvis, thoracic diaphragm, pelvic diaphragm, psoas, and pubic symphysis, were also assessed and diagnosed as deemed necessary by the treating physician (J.P., J.C, C.E., K.B.).

\section{Grayscale US Assessment}

Immediately following diagnosis and immediately after a single OMT session, participants underwent US assessment of their lumbar spine with an Acuson S3000 Ultrasound Scanner (Siemens Medical Solutions). A curvilinear transducer was used to optimize musculoskeletal clarity of transverse processes. Gain and depth were adjusted as needed. All measurements were made with the participant in a prone position using the following settings: scanning frequency, $3.5 \mathrm{MHz}$; image depth, $7 \mathrm{~cm}$; dynamic range, 60; single-image focus; tissue harmonic imaging. All US imaging of the lumbar spine was performed twice by 2 observers ( J.G., who had 30 years of experience performing US, and A.K., who had 2 years of basic training). Each observer took 2 sets of measurements for testing intraobserver variability. The 2 observers performed US separately for testing interobserver variability. The transducer was placed directly over the landmark being measured with only enough applied pressure to achieve satisfactory imaging (Figure 1). The US images were saved, and measurements were performed using the Digital Imaging and Communications in Medicine viewer computer program by multiple observers (J.W., A.K., S.M.) with duplicate measurements made for inter- and intraobserver variability.

To assess the rotational position of each vertebral level (L1-L5), the distance from skin to the most posterolateral surface of the transverse processes was measured $\left(R_{L}\right.$ and $R_{R}$; Figure 2$)$. Distance between adjacent transverse processes (L1-L2) were measured to assess side-bending asymmetries $\left(\mathrm{SB}_{\mathrm{L}}\right.$ and $\mathrm{SB}_{\mathrm{R}}$; Figure 2). Due to the depth of sacral landmarks from the skin, there was difficulty imaging and measuring an accurate distance from L5 to sacrum. For this reason, side-bending measurements were only made

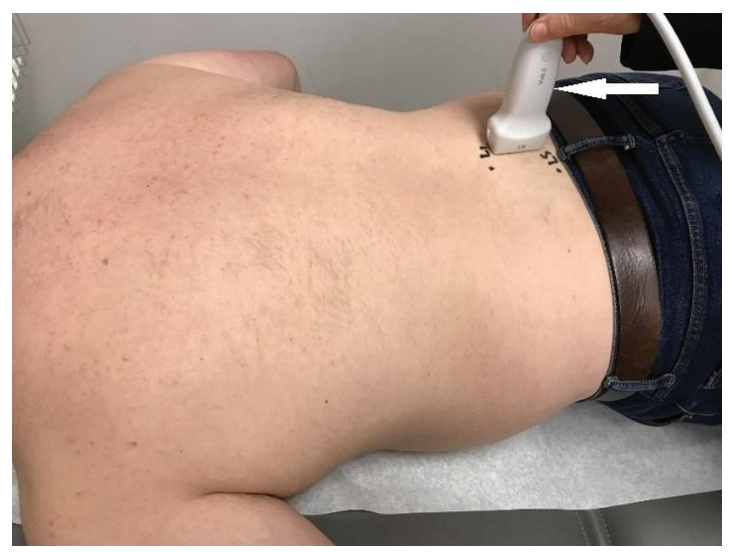

Figure 1.

Ultrasonography (US) of the lumbar spine was performed with the participant prone and with the back muscles relaxed. The transducer (white arrow) was directly placed on the skin of lower back over the transverse processes (L1-L5). Markings were made on the skin by the physician to ensure that US images were recorded at the same vertebral level before and after osteopathic manipulative treatment. These marks were guidelines for the US operators and not specific points of measurement. 


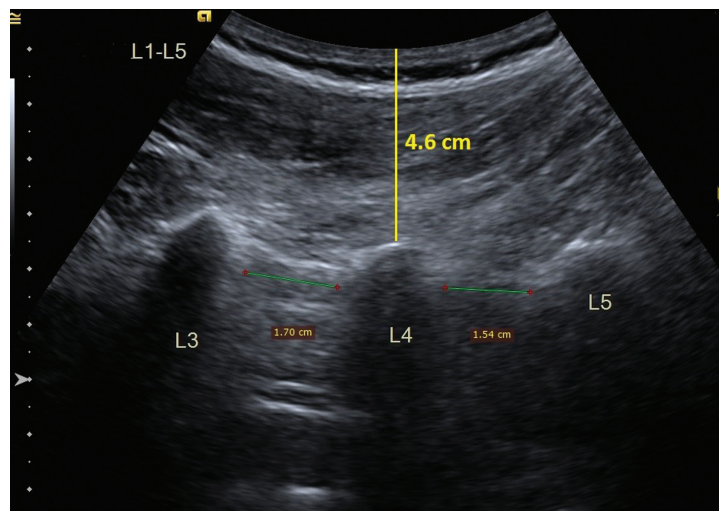

Figure 2.

Grayscale ultrasonography was performed in the low back (L1-L5) region in all participants. Longitudinal static image of transverse processes and intertransverse ligaments were recorded twice by both operators. The anterior-posterior distance (yellow vertical line, $4.6 \mathrm{~cm}$ ) between the skin and the transverse process, and the distance between adjacent transverse processes (green horizontal lines, $1.70 \mathrm{~cm}$ between $L 3$ to $L 4$, and $1.54 \mathrm{~cm}$ between L4 and L5), were measured using electronic calipers online and again offline. Sample measurements were taken at $L_{4}$ on the left side.

for L1 to L4. Rotational and side-bending asymmetry were calculated from these measurements using the following: $\mathrm{R}_{\mathrm{A}}=\left|\mathrm{R}_{\mathrm{L}}-\mathrm{R}_{\mathrm{R}}\right|$ and $\mathrm{SB}_{\mathrm{A}}=\left|\mathrm{SB}_{\mathrm{L}}-\mathrm{SB}_{\mathrm{R}}\right|$.

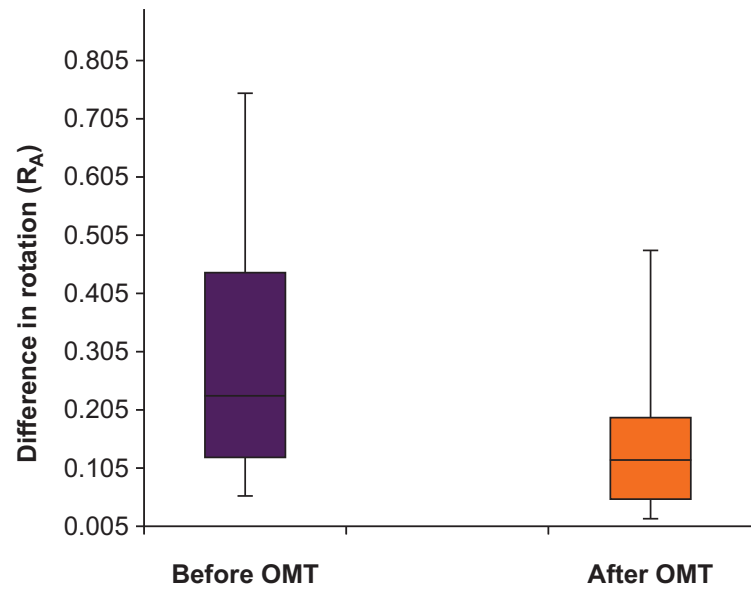

Figure 3.

Box-and-Whisker plots illustrate the difference in spine rotation asymmetry $\left(R_{A}\right)$ before (purple colored box) and after (orange colored box) OMT ( $P=.01)$. A significant decrease in $\mathrm{R}_{\mathrm{A}}$ after OMT indicates the improvement of asymmetry resulting from an effective OMT technique.
All measurements were repeated after the patient received OMT.

\section{Osteopathic Manipulative Treatment}

Following US assessment, participants received a single 15-minute session of OMT to address the previously diagnosed dysfunctions. The techniques used are shown with each participant's corresponding diagnosis in Table 1. The scope of this study did not include evaluating the efficacy of any particular management modality. For this reason, the osteopathic physicians (J.P., J.C, C.E., K.B.) were given instructions to use any modalities they believed would be most effective. OMT was performed with the main goal of improving lumbar asymmetry. Physicians prioritized the management of nonlumbar diagnosis to maximize the improvement of the lumbar dysfunction. Physicians used single or multiple OMT techniques based on the assessment to improve lumbar disorders. Techniques used in this study included: muscle energy technique, myofascial release (MR), balanced ligamentous tension, HVLA, Still technique, articulatory technique, facilitated positional release, and counterstain. The physicians were instructed to treat and reassess the participants.

\section{Statistical Analysis}

All variables, including the thickness of the soft tissue and the distance between transverse processes, were expressed by the mean and standard deviation (SD). Asymmetry was calculated for rotation by measuring the soft tissue thickness from the skin to transverse process bilaterally, and for side-bending by comparing the distance between adjacent transverse processes bilaterally. Calculations were made between the left and right sides of the same segment using the following equations, which we adapted from a previous study: ${ }^{18}$

Rotational asymmetry $=\left|R_{L}-R_{R}\right|$

Side-bending asymmetry $=\left|\mathrm{SB}_{\mathrm{L}}-\mathrm{SB}_{\mathrm{R}}\right|$

$R_{L}$ is the rotational measurement of the left transverse process, and $R_{R}$ is the rotational measurement of 
Table 1.

Lumbar Diagnosis and OMT Technique Used for Each Patient

\begin{tabular}{|c|c|c|c|c|c|c|c|c|}
\hline \multirow{2}{*}{ Patient number } & \multirow{2}{*}{ Lumbar diagnosis } & \multicolumn{7}{|c|}{ Treatment modality used } \\
\hline & & MFR & ME & BLT & HVLA & ST & FPR & ART \\
\hline 1 & L4ESRL & $x$ & $x$ & $x$ & & & & \\
\hline 2 & L1FSRR & $x$ & $x$ & & & & & \\
\hline 3 & L5ESRR & $x$ & $x$ & & $x$ & $x$ & & \\
\hline 4 & T12-L5SLRR & $x$ & $x$ & & $x$ & $x$ & & $x$ \\
\hline 5 & L2-4SLRR & & $x$ & & & $x$ & $x$ & \\
\hline 6 & L1-3SRRL & $x$ & $x$ & $x$ & $\mathrm{x}$ & $x$ & $x$ & \\
\hline 7 & L4FRSR & $x$ & $x$ & $x$ & $\mathrm{x}$ & & & \\
\hline 8 & L5ERSL & $\mathrm{x}$ & $x$ & $x$ & & & $x$ & \\
\hline 9 & L1-5SLRR & $x$ & $x$ & $x$ & $\mathrm{x}$ & & & \\
\hline 10 & L4FRSR & $x$ & $x$ & $x$ & $x$ & & $x$ & \\
\hline 11 & L3ERSL & $x$ & $x$ & & & & & \\
\hline 12 & L5ESRR & $x$ & $x$ & $x$ & & $x$ & & \\
\hline 13 & L3ERSL & & & $x$ & & $x$ & $x$ & $x$ \\
\hline 14 & L3FRSL & & & $x$ & & $x$ & $x$ & $x$ \\
\hline 15 & L4SLRR & $x$ & $x$ & $x$ & & & & \\
\hline 16 & L3SRRL & $x$ & & $x$ & & & & $x$ \\
\hline 17 & L2ESRR & & $x$ & $x$ & & & & \\
\hline 18 & L2-3SRRL & $x$ & $x$ & $x$ & & & & \\
\hline 19 & L4SRRL & $x$ & $x$ & $x$ & $x$ & & & \\
\hline 20 & L3ERSR & $x$ & $x$ & $x$ & & & & \\
\hline
\end{tabular}

Abbreviations: ART, articulatory; BLT, balanced ligamentous tension; HVLA, high velocity low amplitude; CS, counterstrain; FPR, functional positional release; MFR, myofascial release; ME, muscle energy technique; ST, Still technique.

the right transverse process; $\mathrm{SB}_{\mathrm{L}}$ is the measurement between the transverse processes on the left side, and $\mathrm{SB}_{\mathrm{R}}$ is between the transverse processes on the right side. Both asymmetry assessments were measured preand posttreatment.

To assess any change in asymmetry after treatment, pretreatment asymmetry was compared to posttreatment asymmetry using the following equations, which were adapted from a previous study ${ }^{18}$.

Change in rotational asymmetry $=\mid$ Pre- $R_{L}-$ Pre- $R_{R}|-|$ Post- $R_{L}-$ Post- $R_{R} \mid$
Change in side-bending asymmetry $=\left|\mathrm{PreSB}_{\mathrm{L}}-\mathrm{PreSB}_{\mathrm{R}}\right|-$ $\mid$ PostSB $_{\mathrm{L}}-$ PostSB $_{\mathrm{R}} \mid$

Pre- $\mathrm{R}_{\mathrm{L}}$ is the rotational measurement on the left side before OMT and Pre- $R_{R}$ is the rotational measurement on the right side before OMT; and Pre- $\mathrm{SB}_{\mathrm{L}}$ is the intertransverse ligament length on the left before OMT and Pre- $\mathrm{SB}_{\mathrm{R}}$ is the intertransverse ligament length on the right before OMT.

The absolute values allowed us to analyze the group together regardless of the direction of rotation. Without the absolute values, the right and left (positive and 
Table 2.

Lumbar Spine Asymmetry Before and After OMT

\begin{tabular}{|c|c|c|c|c|c|c|c|c|}
\hline \multirow[b]{2}{*}{ Parameters } & \multirow[b]{2}{*}{$\begin{array}{c}\text { Participants, } \\
\mathbf{n}\end{array}$} & \multicolumn{2}{|c|}{$\begin{array}{c}\text { Mean } \\
\text { asymmetry }\end{array}$} & \multirow[b]{2}{*}{$\begin{array}{l}\text { Change in } \\
\text { asymmetry } \\
\text { (cm) }\end{array}$} & \multirow[b]{2}{*}{$\begin{array}{c}\text { Mean \% } \\
\text { improvement }\end{array}$} & \multirow[b]{2}{*}{$\begin{array}{l}\text { Asymmetry } \\
\text { decreased, } n\end{array}$} & \multirow[b]{2}{*}{$\begin{array}{l}\text { Asymmetry } \\
\text { increased, } n\end{array}$} & \multirow[b]{2}{*}{$\begin{array}{c}P \\
\text { value }\end{array}$} \\
\hline & & $\begin{array}{c}\text { Before } \\
\text { OMT } \\
\text { (cm) }\end{array}$ & $\begin{array}{l}\text { After } \\
\text { OMT } \\
(\mathrm{cm})\end{array}$ & & & & & \\
\hline Rotation & 20 & 0.28 & 0.14 & 0.14 & 12.93 & 13 & 7 & 0.01 \\
\hline Side-bending & 16 & 0.23 & 0.21 & 0.03 & -89.07 & 9 & 7 & 0.71 \\
\hline
\end{tabular}

Abbreviation: OMT, osteopathic manipulative treatment.

negative) asymmetries would negate each other and result in a population with no asymmetry. Additionally, greater absolute changes in participants with greater height and mass could introduce bias into our data. We did not have a mechanism to distinguish whether large changes in absolute value were due to the relative size of the participant's anatomy or whether the participants possessed a more significant dysfunction; this is an area for further research. To eliminate this bias, we also represented the changes as mean (\%) improvement for the entire group (Table 2) and the subgroups (Table 3).

Statistical analysis included using an unpaired $t$ test to examine the difference in soft tissue thickness between the sides with and without spinal asymmetry and using a 2-tailed paired $t$ test to analyze the difference in soft tissue thickness before and after OMT. Intra- and interobserver reliability were tested using intraclass correlation coefficient (ICC). The correlation between US findings and osteopathic assessments was analyzed using Pearson correlation coefficient (PCC). A $P$ value less than .05 was considered statistically significant. All statistical analysis was conducted by using SPSS software (SPSS version 25, IBM).

\section{Results}

Data from 20 adult volunteers (10 men and 10 women) were analyzed in this study; US and OMT were successfully performed on all participants. The participant age range was 18 to 55 with a mean age of 31 . Thirteen participants $(65 \%)$ experienced a statistically significant decrease in rotational asymmetry immediately after OMT, as measured by US $(P=.01)$. This improvement in asymmetry suggests that OMT resulted in a less dysfunctional spinal segment. The change in side-bending was not significant after OMT $(P=.71$; Table 2).

Table 3.

Rotation Asymmetry Subgroups

\begin{tabular}{cccccccc} 
& & \multicolumn{4}{c}{ Mean asymmetry, cm } & & \\
\cline { 3 - 7 } & Participants, $\mathbf{n}$ & Before OMT & After OMT & Change in asymmetry & Mean \% change & $P$ value \\
\hline Increased asymmetry & 7 & 0.15 & 0.24 & $-0.08^{\text {a }}$ & $-80.33^{\mathrm{a}}$ & .29 \\
\hline Decreased asymmetry & 13 & 0.35 & 0.09 & 0.26 & 63.15 & .001 \\
\hline & & $P=.02$ & $P=.04$ & & &
\end{tabular}

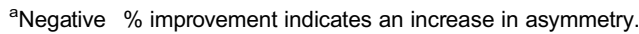


Table 4.

ICC for Online and Offline Ultrasonography Processing for Testing Intra- and Interobserver Reliability

\begin{tabular}{|c|c|c|c|c|c|c|c|}
\hline \multirow{2}{*}{$\begin{array}{l}\text { Comparison between operators } \\
\text { Online }\end{array}$} & \multirow[b]{2}{*}{ ICC } & \multicolumn{2}{|c|}{ 95\% Confidence interval } & \multicolumn{3}{|c|}{$\begin{array}{c}\text { F test with true } \\
\text { value of } 0\end{array}$} & \multirow[b]{2}{*}{$P$ value } \\
\hline & & Lower bound & Upper bound & Value & df1 & df2 & \\
\hline J.G.-J.G. & 0.984 & 0.974 & 0.990 & 63.032 & 63 & 63 & .000 \\
\hline J.G.-A.K. & 0.988 & 0.980 & 0.992 & 81.495 & 75 & 75 & .000 \\
\hline A.K.-A.K. & 0.984 & 0.960 & 0.992 & 80.192 & 75 & 75 & .000 \\
\hline \multicolumn{8}{|l|}{ Offline } \\
\hline A.K.-J.W. & 0.989 & 0.980 & 0.994 & 106.319 & 71 & 71 & .000 \\
\hline A.K.-A.K. & 0.985 & 0.975 & 0.991 & 70.951 & 69 & 69 & .000 \\
\hline J.W.-J.W. & 0.992 & 0.965 & 0.997 & 190.978 & 73 & 73 & .000 \\
\hline
\end{tabular}

Abbreviations: df, degrees of freedom 1; df, degrees of freedom 2; ICC, intraclass correlation coefficient; online, measurements performed using electronic caliper in ultrasonography scanner; offline, all measurements performed on static ultrasonography images using a Digital Imaging and Communications in Medicine reader.

After analyzing the pre-OMT rotational data, we divided the data into subsets based on whether the participant's asymmetry had increased $(n=7)$ or decreased $(n=13)$ after OMT. The 13 participants who showed improvement had significantly more rotational asymmetry before OMT compared with the participants who showed no improvement $(P=.02)$. The group of participants with significant improvement also had significantly less asymmetry after OMT $(P=.04)$.

The changes in absolute value of the depth of the transverse processes were significant for the participants who had a reduction in asymmetry $(P=.001)$. The changes in absolute value of the depth of the transverse processes were not significant for the participants who had increased asymmetry $(P=.29$; Table 3$)$.

To assess the reliability in performing US measurements, we evaluated both intraobserver (J.G.-J.G. ICC $=0.984$, A.K.-A.K. ICC $=0.984$ ) and interobserver ( J.G.-A.K. ICC $=0.988$ ) reliability tests. ICC values greater than 0.9 for both tests demonstrate favorable reliability (Table 4). Additionally, US findings correlated with palpatory assessments of rotational diagnosis ( $\mathrm{PCC}=0.62, P=.004)$, which showed a moderate positive linear relationship between the physician's palpatory assessment and the US findings.

\section{Discussion}

Our results suggest that using 2-dimensional grayscale sonography is feasible to quantify lumbar asymmetry and to assess the efficacy of OMT in the improvement of lumbar asymmetry. US seems to be an effective tool to measure lumbar asymmetry in a neutral position and to monitor the improvement in dysfunctional vertebrae after targeted OMT. A previous study ${ }^{19}$ looked at transverse processes proximal to the vertebral bodies. However, by examining the most posterolateral point of the transverse process, we are able to assess the transverse processes distally, which is the landmark that demonstrates the greatest movement.

We used 2 US operators who were blinded to the osteopathic diagnosis, and 3 observers made US measurements to eliminate measurement bias. Ultimately, we observed favorable intra- and interobserver reliability in performing US - not only in acquiring US images but also in measuring soft tissue thickness and the distance between the most distal portion of transverse processes. ICC results suggested that both online technique and offline processing produced reliable and reproducible identification and measurement of the lumbar landmarks (Table 4). 
When evaluating the sensitivity of US compared with palpation, our PCC results suggested a significant correlation between US and palpatory diagnosis. Research has shown that medical students are capable of palpating asymmetrical differences as small as $4 \mathrm{~mm}$ in models. ${ }^{21}$ A PCC of 0.62 suggests that while palpation may be more sensitive, US may be an effective way to confirm palpatory diagnosis quantifiably. This finding may have important applications in an educational setting when teaching palpation techniques.

The statistically significant decrease in rotational asymmetry suggests that OMT using varied techniques improves lumbar spine somatic dysfunction. Because we used multiple treatment modalities on multiple biomechanically related structures, our data is inconclusive as to which OMT techniques produced the most significant results. Future studies could limit the type of OMT used and the structures treated.

Side-bending asymmetry did not show significant improvements, which may indicate difficulties in assessing this plane of motion either by palpatory or US methods. Additionally, participants who demonstrated significant improvement had more asymmetry before OMT than participants in the group who did not show significant improvement. This finding may indicate that the group that did not improve had less somatic dysfunction present before receiving treatment and were subsequently less affected by the treatment received.

This study was limited by a small sample size, the lack of comparison between asymptomatic participants as a control group and symptomatic patients as a variable group, and the absence of intra- and interobserver reliability tested in performing osteopathic assessments. US was performed immediately after OMT, and the effect of OMT days or weeks posttreatment was not assessed. Future studies should include a larger sample size for greater statistical validity, a control group that receives no treatment and a treatment group of participants who demonstrate symptoms of lower back pain, and dynamic US measurements in addition to static measurements. The longitudinal effects of OMT should also be examined.

\section{Conclusions}

Our results suggest that US can be used as a reliable method to measure somatic dysfunction in the lumbar spine quantifiably. US was also demonstrated to provide a reliable measure of lumbar landmarks and the correlating palpatory diagnosis for the rotational plane of motion, further establishing US as a valuable tool for OMT research. Additionally, our findings support that US can be used to quantify the effects of OMT in restoring symmetry of lumbar spine somatic dysfunctions. Furthermore, our results suggest that a general OMT approach can produce quantifiable statistically significant improvements in lumbar spine rotational asymmetry. Additional research is needed to correlate these findings with varying patient presentations and specific OMT treatments and further examine side-bending dysfunctions.

\section{Acknowledgments}

We thank Siemens Medical Solutions for loaning the ultrasonography scanner used in this study. We also thank Jan Pryor, DO, Judy Caldwell, DO, Chris Edwards, DO, Keith Bodrero, DO, and Amanda O'Loughlin, OMS III, for providing diagnoses, OMT, and technical support to this study.

\section{Author Contributions}

All authors provided substantial contributions to conception and design, acquisition of data, or analysis and interpretation of data all authors drafted the article or revised it critically for important intellectual content; all authors gave final approval of the version of the article to be published; and all authors agree to be accountable for all aspects of the work in ensuring that questions related to the accuracy or integrity of any part of the work are appropriately investigated and resolved.

\section{References}

1. Hoy D, Bain C, Williams $G$, et al. A systematic review of the global prevalence of low back pain. Arthritis Rheum. 2012;64(6):2028-2037. doi:10.1002/art.34347

2. Verhaeghe N, Schepers J, Dun PV, Annemans L. Osteopathic care for low back pain and neck pain: a cost-utility analysis. Complement Ther Med. 2018;40:207-213. doi:10.1016/j.ctim.2018.06.001

3. Licciardone JC, Buchanan S, Hensel KL, King HH, Fulda KG, Stoll ST. Osteopathic manipulative treatment of back pain and related symptoms during pregnancy: a randomized controlled trial. Am J Obstet Gynecol. 2010;202(1):43. doi:10.1016/j.ajog.2009.07.057

4. Licciardone JC, Kearns CM, Minotti DE. Outcomes of osteopathic manual treatment for chronic low back pain according to baseline pain 
severity: results from the OSTEOPATHIC Trial. Man Ther. 2013;18 (6):533-540. doi:10.1016/j.math.2013.05.006

5. Krebs EE, Carey TS, Weinberger M. Accuracy of the pain numeric rating scale as a screening test in primary care. $J$ Gen Intern Med. 2007;22(10):1453-1458. doi:10.1007/s11606-007-0321-2

6. Potter L, Mccarthy C, Oldham J. Intraexaminer reliability of identifying a dysfunctional segment in the thoracic and lumbar spine. J Manipulative Physiol Ther. 2006;29(3):203-207. doi:10.1016/j. jmpt.2006.01.005

7. Bracht MA, Nunes GS, Celestino J, Schwertner DS, França LC, Noronha MD. Inter- and intra-observer agreement of the motion palpation test for lumbar vertebral rotational asymmetry. Physiother Can. 2015;67(2):169-173. doi:10.3138/ptc.2014-16

8. Degenhardt BF, Snider KT, Snider EJ, Johnson JC. Interobserver reliability of osteopathic palpatory diagnostic tests of the lumbar spine: improvements from consensus training. J Am Osteopath Assoc 2005;105(10):465-473.

9. Savva C, Giakas G, Efstathiou M. The role of the descending inhibitory pain mechanism in musculoskeletal pain following high-velocity, low amplitude thrust manipulation: a review of the literature. J Back Musculoskelet Rehab. 2014;27(4):377-382. doi:10.3233/bmr-140472

10. Degenhardt BF, Johnson JC, Fossum C, Andicochea CT, Stuart MK. Changes in cytokines, sensory tests, and self-reported pain levels after manual treatment of low back pain. Clin Spine Surg. 2017;30(6). doi: $10.1097 /$ bsd. 0000000000000231

11. Alvarenga BAP, Fujikawa R, João F, Lara JPR, Veloso AP. The effects of a single session of lumbar spinal manipulative therapy in terms of physical performance test symmetry in asymptomatic athletes: a single-blinded, randomised controlled study. BMJ Open Sport Exerc Med. 2018;4(1). doi:10.1136/bmjsem-2018-000389

12. Sanders GD, Nitz AJ, Abel MG, et al. Effects of lumbosacral manipulation on isokinetic strength of the knee extensors and flexors in healthy subjects: a randomized, controlled, single-blind crossover trial. J Chiropr Med. 2015;14(4):240-248.
13. Haldeman $\mathrm{S}$. The clinical basis for discussion of mechanisms of manipulative therapy. In: Korr IM, ed. The Neurobiologic Mechanisms in Manipulative Therapy. Springer; 1978: 53-75. doi:10.1007/ 978-1-4684-8902-6_4

14. Bialosky JE, Bishop MD, Price DD, Robinson ME, George SZ. The mechanisms of manual therapy in the treatment of musculoskeletal pain: a comprehensive model. Man Ther. 2009;14(5):531-538. doi:10.1016/j.math.2008.09.001

15. Potter L, Mccarthy C, Oldham J. Physiological effects of spinal manipulation: a review of proposed theories. Phys Ther Rev. 2005;10 (3):163-170. doi:10.1179/108331905x55820

16. Savarese RG, Capobianco JD, Adesina AT, Reed G. OMT Review: A Comprehensive Review in Osteopathic Medicine. $4^{\text {th }}$ ed. OMT Review; 2018.

17. Stiffler KA, Jwayyed S, Wilber ST, Robinson A. The use of ultrasound to identify pertinent landmarks for lumbar puncture. Am J Emerg Med. 2007;25(3):331-334. doi:10.1016/j.ajem.2006.07.010

18. Snider KT, Redman CL, Edwards CR, Bhatia S, Kondrashova T. Ultrasonographic evaluation of the effect of osteopathic manipulative treatment on sacral base asymmetry. J Am Osteopath Assoc. 2018;118(3):159. doi:10.7556/jaoa.2018.035

19. Shaw KA, Dougherty JJ, Treffer KD, Glaros AG. Establishing the content validity of palpatory examination for the assessment of the lumbar spine using ultrasonography: a pilot study. J Am Osteopath Assoc. 2012;112(12):775. doi:10.7556/jaoa.2012.112.12.775

20. Habenicht AL. Palpatory Examination. In: Chila AG. Foundations of Osteopathic Medicine. Wolters Kluwer Health/Lippincott Williams \& Wilkins; 2011:401-409.

21. Snider EJ, Pamperin K, Johnson JC, Shurtz NR, Degenhardt BF. Assessing palpation thresholds of osteopathic medical students using static models of the lumbar spine. J Am Osteopath Assoc. 2014;114 (6):460-469. doi:10.7556/jaoa.2014.096

๑) 2020 American Osteopathic Association 